patient's discharge and therefore is a source of morbidity.

It has been our practice in Rochdale to have a running total of wound infections. However, unless this sort of survey is personally supervised the results are likely to be grossly inaccurate as was found to be the case in our hospitals. It is of interest to note that the corrected infection rate in our wounds was $5 \%$ in clean wounds and $27 \%$ in potentially infected wounds, compared with the Aberdeen figures of $5.4 \%$ and $19.7 \%$, respectively. However, only one of our three wards surveyed is subdivided, the others being of Nightingale tvpe, and none has positive-pressure ventilation, the addition of which would undoubtedly be associated with improvement, as was noted from the published series.

In conclusion I would like to make a plea that infection surveys be carried out routinely in peripheral hospitals; the survey must be personally supervised by a consultant (pathologist or surgeon) and is likely to be more effective if performed for a short intensive period rather than a routine affair. The active help and encouragement of the nursing staff and the junior hospital doctors must be sought. Most peripheral hospitals have not the facilities which are available in Aberdeen but this should not preclude meaningful assessment of performance.-I am, etc.,

Birch Hill Hospital

I. G. SCHRAIBMAN

Rochdale, Lancs.

\section{Abnormal Bile or Faulty Gall Bladder?}

SIR,-I enjoyed reading your leading article on the aetiology of gallstones 5 December, p. 571). You rightly quoted the important study of Small and Rapo which showed that in patients with cholesterol gallstones the bile is already overloaded with cholesterol when secreted by the liver. ${ }^{1}$ Essentially the same findings were reported at about the same time and quite independently by Vlahcevic, Bell, and Swell. ${ }^{2}$ Moreover, these workers have gone on to make the important new observation that patients with gallstones have an abnormally small bile-salt pool. ${ }^{3}$ If confirmed, this finding will strengthen the case for the "abnormal bile" origin of gallstones and provide a mechanism for the poor cholesterol-holding capacity of bile.

The fundamental question, which is not answered by these studies, is "Why does the liver secrete abnormal bile?" Two answers are possible: (1) the liver itself is faultv, and (2) the liver is forced to work faultily by outside influences. The editor of another journal has expressed his opinion in a leading article entitled "Gallstones-a new liver disease?"' In so doing he has prejudged the issue and in effect dismissed the possibility that the liver is abnormal in the sense only that it is responding, quite innocently, to abnormal external influences. Your own leading article also omits any reference to external factors, perhaps in the cause of brevity.

I suggest, however, that it is through the study of external-that is, environmentalfactors that the cause of gallstones will be found rather than through studies of the liver or the gallbladder. There is good epidemiological evidence for this belief. At a special meeting on gallstones in Copenhagen in July 1970 (during the 4th World Congress of Gastroenterology) Dr. Nakayama described how in Japan cholesterol gallstones have become much commoner in the last $\mathbf{4 0}$ to 50 years-that is, during the period of increasing Westernstyle affluence. This report confirmed the trend away from pigment stones and towards cholesterol stones which was first noted in city dwellers and people of the higher social classes. ${ }^{56}$ At the same meeting several European experts agreed that during the last war cholesterol gallstones became less common, at least as a clinical problem. Recently there has been a report of increased incidence of gallstones in Italians who emigrate to Australia, ${ }^{7}$ while in affluent Sweden gallstone incidence has risen to unprecedented levels in the last one or two decades. ${ }^{8}$

All these data point to an environmental cause, associated with the modern Western way of life. A dietary cause seems most likely, both on logical grounds and because of the association between cholelithiasis and obesity. ${ }^{9}$ Indeed, Sarles has produced good evidence of excessive calorie intake in gallstone patients ${ }^{10}$ (though some other workers do not agree). Further work in this field is urgently needed but meanwhile it is worth noting the observation made almost 80 years ago by that astute clinician William Osler: "The subjects (having gallstones) are often stout, and usually very fond of starchy and saccharine food". .11

It is more convenient to study organs and body fluids than whole people and their way of life but $I$ believe it is the latter which hold the key to the gallstone mystery.-I am, etc.

Department of Medicine,

K. W. HeatoN

Bristol Royal Infirmary, Bristo Small, D. M.. and Rano, S.. New England
Fournal of Medicine. 1970, 283, 53.
vlahcevic. Z. R., Beil, C., C., and Swell, L., Gastroentcrology, 1970, 59.62 . 6 Vlahcevic, Z. R.. Bell, C. C., Buhac, I. Farrar,
J. T., and Swell, L., Gastroenterology, 1970, 59, New England fournal of Medicine, 1970, 283. 96 Yagi. T. Tohoku fournal of Experimental Medicine, 1960.72. 117.

6 Maki. T., Archives of Surgery, 1961. 82. 599. Wheeler, M., Hills, L. L., and Laby, B., Gut, 1970, 11. 430

8 Nilsson. S.. Acta Chirurgica Scandinavica, 1970 Suppl. 405.

Friedman. G. D., Kannel, W. B.. and Dawber T. R., Fournal of Chronic Diseases, 1966, 19,

10 Sarles. H.. Chabert. C.. Pommeau, Y., Save, E. Mouret. H., and Gérolami. A.. American

11 Osler. W.. The Principles and Practice of Medicine, Eülinburgh, Pentland, 1892

\section{Resuscitation Too Late}

SIR,-Since I retired I have seen with alarm the treatment of some aged relations and friends-for example,

A confused but happy lady, aged 90, cared for by her daughter in a nursing home, having fallen out of bed was sent to hospital to be "pinned." Acute dyspnoea after operation was not treated, anparently for fear she might succumb from effects of drugs. When she died on the fourth day the house surgeon applied external cardiac massage. The daughter, a State-regis tered nurse who was present, asked that she should be left in peace. The reply was "I have my duty to do."

A lady of 89 , nearly blind with glaucoma and unhappy in living with a son-in-law she disliked, having been "saved" from pneumonia almost annually by antibiotics, was found to have an enormous cervical carcinoma. She was sent many miles from all her relations to a hospital where the nursing was of the very best but where radiotherapy did nothing but make her sick and was abandoned. In a hospital nearer home her catheter was removed, and she was allowed to remain 36 hours in great pain while the house physician mumbled about infection and antibiotics. Now she is at home where her merciful doctor is using morphine.

You have my name. I look forward to $\mathrm{my}$ own old age as ever with distaste, but now with dread.-I am, etc.,

Consultant Physician (retired in 1969)

\section{Indications for Hysterectomy}

SIR,-During the past two years 30 out of a total of 1,264 new patients referred to my general surgical outpatient clinics have previously had abdominal hysterectomy. Pathological examinations of the operation specimens in these 30 cases showed that in 12 no abnormality of the pelvic organs was found. In the remaining 18 the following lesions were present: Endometrial or cervical polyps 7; carcinoma of cervix 5; fibroid uteri 3 ; ovarian cyst 2 ; endometriosis 1. The operations have been performed in several hospitals, mostly in N.E. England. In this group of patients, therefore, no pathological lesion was present in $40 \%$ of them, and in a further $23 \%$ lesions (polyps) which could have been dealt with by conservative surgery were present. In $27 \%$ of the cases pathological processes which could not have been removed except by major surgery had been demonstrated. These figures suggest there has been a shift in the indications for abdominal hysterectomy and it would be of interest to know if they represent a typical sample.-I am, etc.,

Newcastle upon Tyne

John K. McCollum

\section{Metformin and Stanozolol \\ in Blood Fibrinolytic Activity}

SIR,-We have previously reported that a combination of phenformin and stanozolol increases the fibrinolvtic activity in the blood. ${ }^{2}$ We have used this treatment for patients with cutaneous vasculitis, Behçets syndrome, and recurrent deep vein thrombosis where the fibrinolytic activity was reduced, and found that it not only increased this activity but produced clinically beneficial results. ${ }^{2} 34$

We have now investigated whether the same effects could be obtained by the administration of a combination of metformin (Glucophage) and stanozolol. Blood fibrinolvtic activity was ectimated by the euglobulin lysis time (E.L.T.) method using an E.L.T. recorder. ${ }^{5}$ Twenty patients divided into two groups of ten each were investigated.

Group 1.-This group consisted of three patients suffering from recurrent deep vein thrombosis and seven patients who had suffered a myocardial infarction with decreased fibrinolytic activity who were treated with phenformin 